\title{
Le nuove frontiere e la cooperazione transfrontaliera
}

La pratica del federalismo svizzero - malgrado le sue caratteristiche evolutive di processo pragmatico e dal basso - risente oggi più che mai dell'inadeguatezza tra l'evoluzione degli spazi funzionali - economici e sociali - in continua ridefinizione, rispetto alla rigidità degli spazi politico-istituzionali.

L'esistenza di nuovi processi di regionalizzazione a scala variabile ha messo in evidenza, in Svizzera prima ancora che in altri paesi, la necessità di superare in qualche modo le frontiere. Infatti, la logica delle frontiere degli stati è quella della frontiera «linea di separazione» tra entità politico-istituzionali diverse, mentre la logica delle comunità e delle economie è quella della "frontiera quale zona di contatto» sia pur tra realtà socio-economiche e culturali diverse (RATTI, REICHMAN 1993).

Sono queste regioni di frontiera (tra stati, ma al limite anche tra cantoni) che sopportano i "costi della noncooperazione», come indica L. SENN (in BRAMANTI, RATTI 1993):

- costi di duplicazione, quando le due regioni confinanti realizzano due infrastrutture parallele (si pensi alle dogane), oppure servizi collettivi (di trasporto, ma anche sanitari o assistenziali) in concorrenza gli uni con gli altri;

- costi da mancato raggiungimento di economie di scala di sistema: esiste una "soglia minima di efficienza" al di sotto della quale il costo di esercizio di reti infrastrutturali (telecomunicazioni, acquedotti, ecc.) non è ottimale. La noncollaborazione tra aree transfrontaliere può impedire il raggiungimento di tale soglia e generare quindi maggiori costi unitari dei servizi di rete, legati al mancato sfruttamento della capacità di erogazione;

- costi da mancato accordo, se le aree transfrontaliere ritardano la ricerca di soluzioni coordinate a problemi comuni (il disinquinamento di un bacino idrico o lacuale di confine), ripercuotendo sui cittadini della zona i negativi effetti sociali che tale ritardo comporta;

- costi da "programmazione avversa", se le scelte di politica economica tendono verso obiettivi opposti che, non mediati o quantomeno discussi, possono condurre ad una reciproca elisione degli effetti positivi delle politiche stesse. Esempi possono essere tratti da diverse politiche del lavoro, da conflittuali politiche ambientali, ecc.;

- costi da diritto di veto, quando le amministrazioni di aree confinanti intralciano vicendevolmente la realizzazione e soprattutto l'utilizzo di infrastrutture o di servizi di interesse comune; ostacolando la mobilità transfrontaliera di merci e di persone, generano costi aggiuntivi per la collettività.

Nel caso svizzero è sentita la necessità per i cantoni e le comunità locali di avere una specie di «politica estera regionale».

In materia di cooperazione transfrontaliera di nuovo il pragmatismo elvetico riesce a precedere nel tempo le formalizzazioni giuridiche.

Cosi, già a partire dall'inizio degli anni '60 si sviluppa la cooperazione transfrontaliera nella Svizzera del NordOvest, a Basilea, successivamente formalizzata, nel 1975, sotto la formula Regio Basiliensis. Ancorata a partire da una commissione tripartita svizzera, francese, germanica, la "Regio» costituisce una delle regioni transfrontaliere più significative d'Europa, estendendosi sull'Alto Reno, tra il Giura, la Foresta Nera e i Vosgi. La «Regio» raggruppa tre territori nazionali: in Germania lo Stadtkreis Freiburg, i Landkreise Emmendingen e in parte quelli di Breisgau-Hochschwarzwald, di Lörrach e di Waldshut; in Svizzera i cantoni di Basilea-Città e Basilea-Campagna e, in parte, di Argovia, Soletta, Berna e Giura; in Francia i dipartimenti dello «Haut-Rhin» e il territorio di Belfort. Anche a Ginevra opera, a partire dal 1973, la Commissione mista consultativa per la regione franco-ginevrina. Nel 1982/85 viene costituita, questa volta sulla base di un accordo interregionale e non più intergovernativo, la Communauté de travail du Jura, che riunisce la FrancheComté e i cantoni di Berna, Vaud, Neuchâtel e Giura. Su questo modello si crea in seguito il Conseil du Léman nel 1987, tra i cantoni svizzeri di Vaud, Vallese e Ginevra e i due dipartimenti francesi dell'Ain e della Haute-Savoie. Anche la regione transfrontaliera attorno al lago di $\mathrm{Co}$ stanza ha fatto oggetto di collaborazioni puntuali tra autorità regionali svizzere, austriache, germaniche e del Liechtenstein, che probabilmente daranno luogo a un'Associazione di collaborazione regionale meglio formalizzata.

Tra la Svizzera e l'Italia è il Ticino il cantone più interessato, con Vallese e Grigioni, alla cooperazione transfrontaliera. Un primo accordo tra regione Lombardia e cantone Ticino in materia di trasporti data del 1980; il 15 giugno 1990 l'accordo evolve verso una dichiarazione d'in-

Remigio Ratti, Prof. Dr, consigliere nazionale, Istituto di Ricerche Economiche (IRE), Stabile Torretta, 6501 Bellinzona 
tenti per l'istituzionalizzazione di gruppi di lavoro estesi alle materie della pianificazione del territorio, dei trasporti e delle comunicazioni, della protezione della natura, delle acque e dell'aria, della cultura e dell'insegnamento cosi come del mercato del lavoro e di certe attività economiche.

In sostanza i fatti precedono un importante accordo-quadro tra la Confederazione svizzera e la Repubblica italiana per la cooperazione transfrontaliera delle collettività ed autorità regionali e locali, firmato a Berna il 24 febbraio 1993 ed entrato in vigore il 26 aprile 1993.

In particolare per quel che riguarda il territorio nordlombardo-ticinese il 1995 è stato caratterizzato della costituzione di una regio insubrica o "regione dei tre laghi» (il Lario, il Ceresio ed il Verbano). Promossa da un'iniziativa del sindaco di Chiasso, città di frontiera, sulla falsariga della "Regio Basiliensis» dovrebbe dare impulso e accelerare una collaborazione che chiede un approfondimento non solo nel senso del coordinamento ma anche di una progettualità comune.

Il programma europeo «Interreg» va pure in questa direzione stimolando le regioni di frontiera appartenenti all'Unione europea a elaborare progetti in comune anche con regioni di frontiera appartenenti a paesi terzi. La legge italiana 1990/142 sulla riforma dei poteri delle autorità locali prevede del resto lo strumento della Dichiarazione d'intenti e dell'Accordo di programma che dovrebbero facilitare di molto le applicazioni concrete. La regione insubrica, costruita attorno ai tre poli di Como, Varese e Lugano, costituisce una regione a carattere transfrontaliero in senso stretto (fascia di $20 \mathrm{~km}$ dal confine), con 1,2 milioni di abitanti, le cui strutture economiche e sociali hanno subito un processo evolutivo secondo logiche contrapposte, mentre potenzialmente si tratta di un'area che presenta delle interessanti complementarità da mettere in risalto (cfr. BRAMANTI, RATTI 1993).

Questa costruzione di uno spazio economico transfrontaliero costituisce un processo di nuova territorializzazione che mette le periferie, le regioni di frontiera, al centro di un processo che appare ineluttabile all'interno del Mercato Unico Europeo. Questa nuova interpretazione della frontiera come spazio di contatto non deve essere considerata come una forza centrifuga rispetto allo statonazione ma, al contrario, può contribuire al rafforzamento dell'armatura territoriale regionale. Assistiamo al sorgere di nuove aggregazioni regionali metanazionali che, senza distruggere quelle che sono le eredità storiche e le appartenenze ai "vecchi stati nazionali», assumono grande significato culturale, sociale ed economico. In particolare l'apporto del Ticino alla Svizzera potrà essere migliore in un nuovo contesto, allargato alla cooperazione transfrontaliera e quindi in uno scenario che non lo vede più doppiamente periferico e marginalizzato rispetto all'oltralpe e al suo spazio culturale italiano.

Oltre alla collaborazione transfrontaliera di vicinato non bisogna dimenticare la collaborazione transfrontaliera a scala interregionale, per esempio quella dell'Arco alpino.
Anch'essa rappresenta bene la necessità odierna e del futuro di riscoprire nuove forme di territorialità, intesa come capacità di gestione di un sottosistema e quindi come necessità di trovare coerenze nel rapporto "globalelocale».

Sin dal 1973 il «Comitato d'iniziativa per la cooperazione fra le regioni dell'Arco alpino" presieduto dall'allora primo presidente della regione Lombardia, Piero Bassetti, aveva chiaramente intuito che le Alpi non possono e non hanno mai rappresentato una barriera-linea di divisione tra nord e sud Europa, bensi un' area d'intensi contatti con vallate che hanno dato luogo ad una cultura della gestione dal basso e ad autonomie locali potenzialmente ricche perché aperte (AA.VV. 1988). La costituzione, nel 1973, della Comunità di lavoro delle regioni dell'Arco alpino centrale (Arge Alp), poi nel 1978 quella della Comunità di lavoro delle Alpi orientali (Alpe Adria) e quella, nel 1982, della Comunità di lavoro delle regioni e cantoni delle Alpi occidentali (Cotrao) hanno in parte formalizzato e dato inizio alla collaborazione a questa dimensione. In particolare l'Alpe Adria, che ha anticipato il fenomeno delle nuove realtà dell'Europa centrale, comprende oggi 18 regioni di paesi appartenenti all'UE, all'AELS e all'Europa centrale (4 regioni italiane, 5 Länder austriaci, la Baviera, le Repubbliche di Slovenia e Croazia, 5 province ungheresi e, recentemente, il cantone Ticino) interessando un territorio di $284000 \mathrm{~km}^{2}$ e oltre 38 milioni di abitanti.

Da parte sua anche l'Associazione delle regioni d'Europa (ARE), creata nel 1985-1988, e che conta oggi oltre 300 regioni dell'Europa occidentale e centrale, rappresenta, a un'ulteriore scala significativa, la necessità per l'Europa di consolidare una unità che sia alimentata nel contempo dalla forza e dalla diversità delle regioni.

Questa cooperazione transfrontaliera a più livelli si trova ancora in una fase embrionale, e presenta sovente sovrapposizioni ed incertezze operative. Siamo veramente, in questo ambito, in una fase esplorativa e transitoria del riassetto territoriale, il quale implica un lungo discorso e il dialogo con gli stati-nazione e con le nuove istituzioni e istanze europee, anch'esse in una fase di riconfigurazione.

Anche in questo caso la Svizzera ha seguito una via piuttosto pragmatica e dipendente dal grado di iniziativa dei cantoni: la Confederazione ha lasciato fare proprio nel rispetto delle sovranità cantonali, sia pure relativizzate dalla Costituzione federale. Il peso concreto di queste iniziative di collaborazione transfrontaliera orizzontale e verticale alle varie scale - di vicinato, interregionali, ed europee - si presta a valutazioni molto eterogenee e, in genere, è percettibile significativamente solo da chi abbia una particolare sensibilità alla lettura delle dinamiche spaziali e della gestione della territorialità. Il carattere emergente di queste dimensioni ne fanno però un germe delle tendenze di domani, indubbiamente importante in quel processo che porterà a «reinventare la Svizzera» del ventunesimo secolo. 


\section{Bibliografia}

AA.VV. (1988): Le Alpi per l'Europa: una proposta politica, Milano.

BRAMANTI, A., RATTI, R. (1993): Verso un'Europa delle Regioni. La cooperazione economica transfrontaliera come opportunità e sfida, Franco Angeli, Milano.
RATTI, R. (1995): Leggere la "La Svizzera». Saggio politicoeconomico sulle origini e sul divenire del modello elvetico, ISPI, Istituto per gli studi di politica internazionale, Milano/ Giampiero Casagrande Editore, Lugano.

RATTI, R., REICHMAN, S., (a cura di) (1993): Theory and practice of transborder cooperation, Helbing \& Lichtenhahn, Basel/Frankfurt am Main.

\section{Grenzregionen: Außenschweiz und Innereuropa}

\section{Vom Sonderfall Schweiz zum Sonderfall Grenzregionen?}

An den «Sonderfall Schweiz» haben wir uns längst gewöhnt. War «Sonderfall» zur Zeit der selbstzufriedenen Willensnation durchwegs positiv gemeint, schwingt heute - vor allem nach dem Nein zum Europäischen Wirtschaftsraum - doch eher ein negativer Klang («Sorgenfall»?) mit. Wir fragen uns inzwischen, ob die Bezeichnung «Sonderfall» im Zeitalter der zunehmenden Globalisierung von Gesellschaft, Wirtschaft und Umweltproblemen noch ihre Berechtigung hat. Dieselbe Bezeichnung wenden wir aber weiterhin für die Grenzregionen an, meist ohne uns zu fragen: "Sind Grenzregionen ein besonderer (Fall) von Regionen? Haben sie spezifische Regionalprobleme? Wenden die Grenzregionen andere regionalpolitische Strategien als Binnenregionen an?» In diesem Artikel sollen schwergewichtig Regionalpolitiken der Grenzregionen skizziert werden. Eine Vergleichsmöglichkeit - und somit die Basis zur Beantwortung der übrigen Fragen - bietet das vorliegende Themenheft.

\section{Föderalismusreform}

\section{als theoretische Antwort auf Grenzprobleme}

Grenzregionen, genauer subnationale Gebietskörperschaften an den Landesgrenzen, «leiden» theoretisch an ähnlichen Problemen wie die inneren Randregionen:

- Der Kreis der Nutznießer stimmt nicht mit dem Kreis der Kostenträger überein (z. B. Tourismus im ländlichen Raum oder staatlich subventionierte Kulturdienstleistungen in Zentralorten).
- Der Kreis der Geschädigten und der Kostenträger deckt sich ebenfalls nicht mit demjenigen der Verursacher (beispielsweise im Grenzpendler- und kleinen Einkaufsverkehr, wo die Luftverschmutzung und die Lärmbelastungen keine Grenzen kennen. Auch Löschwasserkatastrophen wie der Sandoz-Brand bei Basel sind grenzüberschreitend).

- Politische und wirtschaftliche Entscheidungen fallen häufig außerhalb des Kreises der Betroffenen. So wird die zentralstaatliche Einheit gewahrt. Unliebsame, risikoreiche Anlagen werden aus dem Blickfeld an den Rand des Territoriums verdrängt. In Kauf genommen werden dafür eine gewisse Bürgerferne der Entscheidungen sowie höhere Kontrollkosten in der Peripherie.

- Da in den Grenzregionen die regionalen Grenzen mit nationalen Grenzen zusammenfallen, sind viele Austausche zwischen Nachbarn gar nicht realisierbar. Größenvorteile und komparative Kostenvorteile lassen sich daher nur beschränkt grenzüberschreitend nutzen. Fehlende Produktionen von kollektiven und öffentlichen Gütern oder unausgelastete Verdoppelungen der Produktionen beidseits der Grenze mit hohen Effizienzverlusten sind die Folge.

Solchen Spillovers sowie Problemen der Kleinheit und Problemen der allzu starken nationalstaatlichen Zentralisierung kann mit einer Föderalismusreform entgegengetreten werden. Im Idealfalle vereinigt der Föderalismus Vorteile der Dezentralität und der Zentralität in

Maria Lezzi, Dr., REGIO BASILIENSIS, Peter-Merian-Straße 21, 4052 Basel 